\title{
Transmission Electron Microscopy Analysis of Defects in Boron Carbide Nanowires
}

\author{
T. Gutu, Z. Guan, and T. Xu \\ Department of Mechanical Engineering and Engineering Science, The University of North \\ Carolina, Charlotte, NC 28223
}

Boron carbide $\left(\mathrm{B}_{4} \mathrm{C}\right)$, a mostly $p$-type semiconductor, exhibits unusual novel electrical and thermal transport properties due to its unique icosahedral-based structure and bonding of the icosahedra. The transport properties are sensitive to the presence of crystallographic defects. For example, twins could cause remarkable increase in the Seebeck coefficient of $\mathrm{B}_{4} \mathrm{C}$-type materials [1]. Though there have been some reports on Transmission Electron Microscopy (TEM) study on planar defects in bulk boron carbide samples and boron carbide whiskers,[2] there is no detailed report in boron carbide nanowires discussing both the longitudinal planar defects (i.e., twin defects parallel to the growth direction of a nanowire) and the transverse planar defects (i.e., twin defects perpendicular to the growth direction of a nanowire). In this report, we show the presence of both longitudinal and transverse planar defects in boron carbide nanowires. The defected nanowires are good candidates for fundamental transport properties studies to reveal unique structure-property relation.

Boron carbide nanowires were synthesized by nickel-assisted co-pyrolysis of diborane $\left(\mathrm{B}_{2} \mathrm{H}_{6}\right)$ and methane $\left(\mathrm{CH}_{4}\right)$ at a high temperature of about $1050{ }^{\circ} \mathrm{C}$ and a low pressure of about 1300 mTorr. A JEOL $2100 \mathrm{LaB}_{6}$ Transmission Electron Microscope was utilized to analyze defects in as-synthesized boron carbide nanowires.

Fig. 1a-d shows TEM images for a nanowire (NW) with transverse planar defects. The Ni catalyst is visible at the tip of the NW in (a). The HRTEM image in (b) clearly reveals the twins/stacking faults and the red line indicates the existence of these effects. The streaking in the corresponding diffraction pattern in (c) indicates the high density of the planar defects. The dark field image, in (d), formed by selecting diffracted beams from the streaking shows the defect planes as bright/dark lines that are normal to the NW [101] growth direction. Fig. 1e-h shows the corresponding images for a NW with longitudinal planar defects. Images (f)-(h) reveal that the density of the planar defects in this NW is low. In fact, the streaking is evidently absent in the diffraction pattern in $(\mathrm{g})$. Fig. $1 \mathrm{~h}$ reveals that the planar defects are confined to one half of the NW.

[1] D. Emin, Physics Today, 40, (1987), p55.

[2] I.D. Mackinnon, T. Aselage and S. B. Van Deusen, AIP Conf. Proc. 140, (1985), p114. 

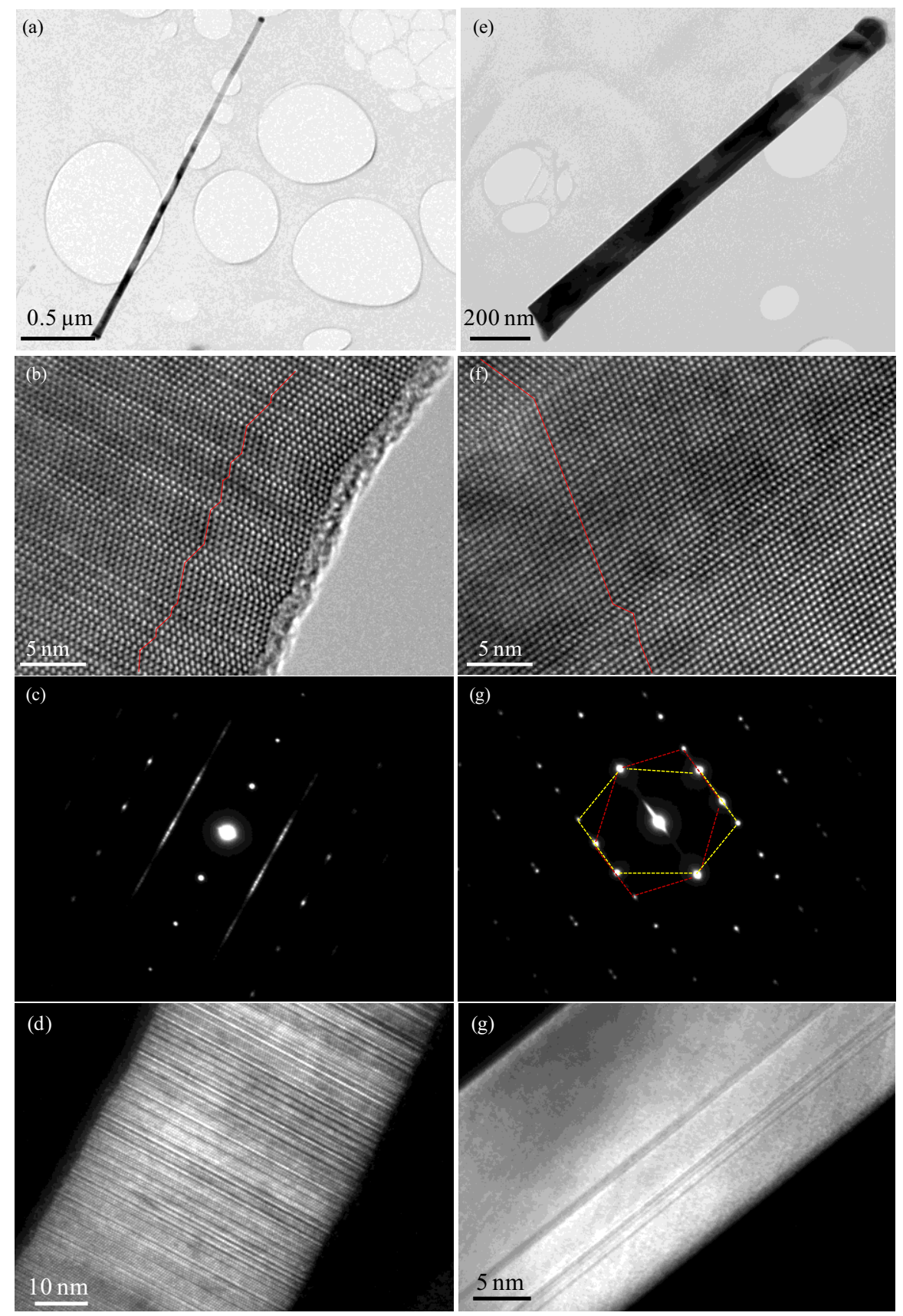

Fig. 1 Two B4C nanowires: (a-d) Twin planes perpendicular to the growth direction of a nanowire, (a) low magnification bright field image, (b) HRTEM image, (c) corresponding diffraction pattern and (d) dark field image; (e-g) twin planes parallel to growth direction, (e) low magnification bright field image (f) HRTEM image, (g) corresponding diffraction pattern, reflections from regions indicated by A and B in (f) are shown by yellow and red dashed lines respectively and (h) dark field image. 